\title{
Effects of Dietary n-3/n-6 PUFA Ratios on Animal Behaviors and Inflammation in a Prenatal Valproic Acid Exposure-Induced Rat Model of Autism
}

\author{
Yuxi Jia ${ }^{1,2}$, Ping $\mathrm{Li}^{3}$, Fei $\mathrm{Wu}^{4}$, Fanjie Zeng ${ }^{3}$, Beilin Zhang ${ }^{5}$, Jinpeng Wang $\mathbb{1}^{6}$ \\ 'Application Demonstration Center of Precision Medicine Molecular Diagnosis, The Second Hospital of jilin University, Changchun, I3004I, People's \\ Republic of China; ${ }^{2}$ Department of Orthopedics, The Second Hospital of Jilin University, Changchun, I 3004I, People's Republic of China; ${ }^{3}$ Department \\ of Developmental Pediatrics, The Second Hospital of Jilin University, Changchun, I 3004I, People's Republic of China; ${ }^{4}$ Department of Gynecology and \\ Obstetrics, the Second Hospital of jilin University, Changchun, 13004I, People's Republic of China; ${ }^{5}$ Department of Physiology, College of Basic Medical \\ Sciences, Jilin University, Changchun, I 3002 I, People's Republic of China; ${ }^{6}$ Department of Cardiology, the Second Hospital of jilin University, Changchun, \\ I3004I, People's Republic of China
}

Correspondence: Jinpeng Wang, Email jinpeng0865@sina.com

Background: In clinic, valproic acid (VPA) has been widely used in the treatments of epilepsy and is currently under clinical trials for the treatments of multiple diseases. However, gravidas receiving VPA treatment during their early stage of pregnancy were reported to be associated with elevated incidences of autism in their child. In this study, the potential influences of n-3/n-6 polyunsaturated fatty acids (PUFAs) as supplements were investigated in VPA-induced autistic rat model.

Materials and Methods: A rat model of autism spectrum disorder (ASD) was established by VPA exposure at the E12.5 day of gestation. Neonatal male Wistar rats $(\mathrm{n}=24)$ were divided into VPA group, A group and B group, while normal neonatal male Wistar rats served as controls. At P21 days, animals in the control and VPA groups were fed with standard chow, while rats in the A group were fed with n-3 PUFA free chow, and those in the B group with n-3/n-6 PUFA (1:5) chow. The duration of the diet was from P2 1 days to 3 months. All rats were subjected to the examinations of repetitive stereotyped behaviors and changes in social interaction at the end of supplement. The neuroinflammation in the brain and serum was also evaluated. Hematoxylin-eosin (HE) staining and Nissl staining were used to observe the neuronal injury in hippocampus.

Results: In the B group, the repetitive stereotyped behaviors reduced, the social communication was improved, the neuronal loss and proinflammatory cytokines in the hippocampus reduced, and the extracellular STAT3 activation was inhibited in the hippocampus, as compared to the B group.

Conclusion: Supplements with n-3/n-6 PUFA (1:5) rescues repetitive stereotyped behaviors and social deficits in neonatal rats exposed to VPA, likely due to the improved inflammatory responses through the alteration of STAT3 signaling. This study provides evidence for the use of n-3/n-6 PUF in the treatment of autism in humans.

Keywords: polyunsaturated fatty acids, autism spectrum disorder, valproic acid, hippocampus, inflammation

\section{Introduction}

Autism spectrum disorder (ASD) is a neuropsychiatric condition caused by abnormal neurodevelopment that can impact the perception and socialization of a person. Symptoms of ASD include restricted interests, impaired social interaction due to stereotyped, repetitive behaviors, and communication deficits. ${ }^{1}$ However, the etiology of ASD is not clear.

Valproic acid (VPA) has been used for the treatment of epilepsy, bipolar disorders and migraine. VPA is able to regulate the activities of some genes, cause damages to DNA, and modulate the mitochondrial energy metabolism, due to its histone deacetylase inhibitory and epigenetic modulation activities. VPA is also demonstrated to elevate the oxidative stress in fetus, therefore acting as a potential teratogen. Some symptoms and their severities are related to the fetal valproate syndromes, including defections in the development of neural tube, neurodevelopmental delay, dysmorphic features, cardiac anomalies, cognitive defects and ASD-related features. ${ }^{2}$ 
Previous studies have revealed that VPA treatment on gestational 12.5th day causes changes in various behaviors as well as molecular and neuroanatomical features in the offspring rats similar to the clinical symptoms of ASD patients. Those changes normally include impaired social behaviors, anxiety disorders, and hyperactivities in locomotive and repetitive behaviors of rats. ${ }^{3}$ Preclinical studies have indicated that ASD is associated with the Purkinje cells loss in the cerebella, cranial nerve motor nuclei damages, brainstem neurons reduction and increase of proinflammatory cytokines, including interleukin-6 (IL-6) and tissue necrosis factor-alpha (TNF- $\alpha$ ), in the brain. ${ }^{4-6}$

ASD has a robust association with altered lipids in the neuronal processes and immunological responses. Polyunsaturated fatty acids (PUFAs) are essential for the neurodevelopment and maturation of the central nervous system (CNS). ${ }^{7}$ Omega-3/omega-6 (n-3/n-6) PUFAs, specifically, n-3 PUFA docosahexaenoic acid (DHA) and n-6 PUFA arachidonic acid (AA), are enriched in the CNS. ${ }^{8} \mathrm{DHA}$ is necessary for normal functions of the retina and synapses, and participates in the regulations of neurotransmitters. Recent studies revealed that the consumption of n-3 PUFAs is declining in the current diet. The approximate n-6/n-3 PUFAs ratio is up to around 16:1 in modern Western diet, which is significantly higher than that in ancient Western diet (1:1). As a result, the excess $n-6$ PUFAs displace the position of $n-3$ PUFAs on the cellular membranes. This altered ratio of n-6/n-3 PUFAs therefore results in the increase of inflammatory reactions, thrombosis and vasoconstrictions due to the conflict functions of eicosanoids derived from arachidonic acid (AA) and eicosapentaenoic acid (EPA), which are respectively belong to $n-6 / n-3$ PUFAs. ${ }^{9}$

Recent studies have indicated the alteration in the n-3 PUFA homeostasis of ASD panties. However, whether this alteration is caused by nutritional imbalance or genetic defection is still not clear. ${ }^{10}$ There is evidence showing that the depletion of n-3 PUFA during the development of rodents causes serotonin decrease in the prefrontal cortex, which is also observed in ASD children. ${ }^{11}$ DHA is effective and beneficial in the cognitive development in the infants with preterm birth. Keim et al found that in clinical random tests, children and juniors receiving omega-3-6-9 supplements showed statistically significant improvements in ASD-related symptoms, which provide evidence for potential clinical use and safety assurance of PUFAs supplements in large-scale trials on ASD. ${ }^{12}$ Puri and Maratins ${ }^{13}$ reported that treatment with both EPA and $\gamma$-linolenic acid (GLA) at 18:3 showed the highest efficiency in the treatments of behaviors associated with attention-deficit-hyperactivity disorders.

Recently, the growing prevalence and poor therapeutic efficacy of ASD draw world-wide attention. Herein, this study was undertaken to investigate the potential effects and molecular mechanisms of PUFA at different n-3/n-6 ratios on the ASD in rats exposed to VPA prenatally.

\section{Materials and Methods Experimental Design}

All the procedures were performed according to the National Animal Care Guidelines, and this study was approved by the Ethics Committee of Jilin University (No. 2019071). Wistar rats were housed in a standard environment $\left(22 \pm 2{ }^{\circ} \mathrm{C}\right.$, 50-60\% humidity, $12 \mathrm{~h} / 12 \mathrm{~h}$-light/dark cycles). Rats (female: male=2:1) were grown in cages overnight. The next morning, each female rat was checked for the vaginal plug, which indicates successful pregnancy. The day of vaginal plug formation was defined as embryonic day 0.5 (E0.5). Pregnant rats were separated into VPA-reated group and control group. Pregnant rats were treated with VPA (Aladdin, Shanghai, China; $600 \mathrm{mg} / \mathrm{kg}$ ) through intraperitoneal (i.p.) injection. Aliquots of sterile saline were used for controls. Only male offspring rats were subjected to subsequent experiments. Rats were examined for symptoms of ASD-related behaviors. Rats with established ASD were subsequently divided into autism group with only VPA treatment (VPA group), autism with n-3 PUFA insufficient group (A group) and autism+n-3/n6 PUFA=1:5 supplements (B group). The animal behaviors were evaluated at 3 months after birth.

\section{Diet}

All offspring rats were weaned 21 days after birth and fed with standard rodent chow. Meanwhile, rats in A group were fed with standard rodent chow + n-3 free supplements. Rats in B group were fed with standard rodent chow + n-3/n- 6 PUFA (1: 5) supplements. Food composition was the same as described in our previous experiment (Table 1). ${ }^{14}$ Diet was administered from the 21st day after birth to 3 months. 
Table I Diet Macronutrient Profiles

\begin{tabular}{|l|c|c|c|}
\hline Ingredients (g/kg) & Control & A (n-3 free) & B (n-3/n-6=I:5) \\
\hline Casein & 200 & 200 & 200 \\
Corn starch & 388 & 388 & 388 \\
Sucrose & 150 & 150 & 150 \\
Methionine & 3 & 3 & 3 \\
Sunflower seed oil & 0 & 660 & 110 \\
Vitamin mixture & 10 & 10 & 30 \\
Mineral mixture & 35 & 35 & 47 \\
Cellulose & 47 & 47 & 2.5 \\
Choline & 2.5 & 2.5 & 4 \\
Calcium & 4 & 4 & 300 \\
Lard & 0 & 300 & 40 \\
Linseed oil & 0 & 0 & 510 \\
Fish oil & 0 & 0 & 0 \\
\hline
\end{tabular}

\section{Behavioral Tests}

Social Play Behaviors

Social play behaviors of rats were observed as described previously. ${ }^{15}$ Behavior observation was carried out in noiseproofed chambers under dimly lit environments. Rats were placed in Plexiglas cages $(1 \times \mathrm{w} \times \mathrm{h}: 40 \times 40 \times 60 \mathrm{~cm})$ covered with sawdust bedding. The behaviors of rats were recorded for further analysis. Two days before the test, each rat was placed into the test environment for $10 \mathrm{~min}$ for 2 consecutive days for adaptation. Rats were housed insolently for $3 \mathrm{~h}$ prior to the test in order to promote the social motivations, and therefore enhance the social play behavior expressions during tests. ${ }^{16}$ During the test, two rats were subjected into one group, and placed in the same test cage for $15 \mathrm{~min}$. Rats were paired based on two criteria: body weight difference within $10 \mathrm{~g}$; no previous common social experience. The following parameters were scored: 1) Frequencies of pouncing: temptation of nosing or rubbing between paired rats and 2) Time spent on social explorations: sniffing of body parts (including the anogenital area) between paired rats.

\section{Self-Grooming Behaviors}

Self-grooming test paradigm was measured to reflect the stereotyped behaviors of rats. ${ }^{17}$ Rats were placed into a new empty cage and left alone for habituation for $10 \mathrm{~min}$. Subsequently, spontaneous behaviors of each rat were recorded for $10 \mathrm{~min}$ and analyzed for accumulative self-grooming time. In this study, features of self-grooming behaviors include 1) wiping of body with forepaws and 2) licking of body parts including whole body, anogenital area and tail.

\section{Sample Collection}

Rats were intraperitoneally injected with 3\% sodium pentobarbital for anesthesia and killed immediately by decapitation. Blood samples were collected freshly and centrifuged to collect serum. Whole brain tissue of rats was collected freshly and cut into coronal slices. Whole process was carried out on ice. The hippocampal tissue (HIPP) was harvested under a microscope within 2 min as previously described. ${ }^{18}$

\section{Enzyme-Linked Immunosorbent Assay (ELISA)}

Rat hippocampal tissues $(50 \mathrm{mg}$ ) were homogenized in $500 \mu \mathrm{L}$ of tissue protein extraction buffer (Beijing CoWin Biotech) on ice. The resulting homogenates were subjected to centrifuge at $10,000 \times \mathrm{g}\left(4{ }^{\circ} \mathrm{C}, 20 \mathrm{~min}\right)$. Protein concentration was determined with BCA Kit (Beijing CoWin Biotech). Hippocampal concentrations of interleukin-1 (IL-1), IL-6 and tumor necrosis factor- $\alpha$ (TNF- $\alpha$ ) were measured with corresponding ELISA Kits (IL-1: No.368191224; IL-6: No. 385191224; TNF- $\alpha$ : No. 569191224) (Anoric Bio-technology, Tianjin, China). Concentrations of IL-1, IL-6 and TNF- $\alpha$ were calculated according to corresponding standard curves and normalized to total protein concentrations. 


\section{Hematoxylin-Eosin (HE) Staining and Nissl Staining}

After behavioral tests, rats were anesthetized and cardiovascular perfused using 0.1 PBS (pH 7.4) and 4\% paraformaldehyde dissolved in PBS. Whole brain tissues were harvested and incubated in $4 \%$ paraformaldehyde at $4{ }^{\circ} \mathrm{C}$ for $48 \mathrm{~h}$, and subsequently embedded into paraffin. Slices $(10 \mu \mathrm{m}$ every $20 \mu \mathrm{m})$ were cut throughout the brain. Sections containing hippocampus were subjected to HE staining. In Nissl staining, slices $(4 \mu \mathrm{m})$ were obtained from the formaldehyde-fixed brain (embedded in paraffin) and incubated with staining solution for $5 \mathrm{~min}$. Neurons with lesions were found to be shrunken or contain vacuoles. Meanwhile, the normal neurons presented relatively larger soma with round and large nuclei. Numbers of neuron were averaged in three rats at the same section of the brain in each group. Results were analyzed by Image J and evaluated in a blind manner.

\section{Western Blotting}

Samples were extracted from hippocampal tissues. Protein concentrations were determined by bicinchoninic acid protein assays (Biotype Biotech Co., Shanghai, China). Total protein was fractionated by $12 \%$ SDS-PAGE electrophoresis and transferred onto polyvinylidene difluoride (PVDF, 0.22- $\mu \mathrm{m}$ ) membrane. Then, the membrane was blocked in 5\% skimmed milk for $2 \mathrm{~h}$ at room temperature $\left(23-25{ }^{\circ} \mathrm{C}\right)$. Primary antibodies used were as follows: STAT3 (bsm52235R, weight $=88 \mathrm{kDa}, 1: 1000$, Bioss Biotechnology, Beijing, China) and GAPDH (bs-2188R, 1:1000, Bioss Biotechnology, Beijing, China). Membranes were washed thrice with TBST and then incubated with horseradish peroxidase (HRP)-conjugated secondary antibody (bs-0295g-HRP, 1:10,000; Bioss Biotechnology, Beijing, China).

\section{Statistical Analysis}

All experiments were carried out in triplicates. Data were subjected to tests for homogeneity and normality. Statistical analysis was carried out with SPSS version 17.0 (SPSS, Inc., Chicago, IL, USA) and GraphPad Prism 8 software (GraphPad Software Inc., LaJolla, CA, USA). One-way analysis of variance (ANOVA) and least significant difference (LSD) were employed for comparisons among groups. A value of $\mathrm{P}<0.05$ was considered statistically significant. Data are expressed as mean \pm standard error (SEM).

\section{Results}

\section{Effects of n-3/n-6 PUFA Ratio in Dietary Supplement on Sociability Behaviors of Experimental Autism Rats}

In social play behavior tests, the sniffing frequencies in rats of B group $(18.12 \pm 1.87)$ were significantly higher than in the VPA group (6.88 $\pm 0.91, \mathrm{P}<0.01, \mathrm{n}=8)$. Meanwhile, the sniffing frequencies reduced significantly in the rats of VPA group $(6.88 \pm 0.91)$ as compared to the control group $(14.75 \pm 2.69, \mathrm{P}<0.05, \mathrm{n}=8$, Figure $1 \mathrm{~A})$. The pouncing frequency was comparable between offspring rats with prenatal VPA exposure and rats in the control group (Figure $1 \mathrm{~B}, \mathrm{P}>0.05, \mathrm{n}=8$ ).

\section{Effects of n-3/n-6 PUFA Ratio in Dietary Supplement on Self-Grooming Behaviors of Experimental Autism Rats}

In this study, the average durations of self-grooming were significantly longer in prenatal VPA exposure rats (216.62 $\pm 24.20 \mathrm{~s})$ as compared to control rats $(128.34 \pm 29.92 \mathrm{~s}, \mathrm{P}<0.01, \mathrm{n}=8)$. Meanwhile, the average durations of self-grooming time in n-3/n-6 PUFA (1:5)-supplemented rats (157.90 $16.73 \mathrm{~s})$ decreased markedly as comparing to the prenatal VPA exposure rats (Figure 2, $\mathrm{P}<0.05, \mathrm{n}=8$ ).

\section{Effects of n-3/n-6 PUFA Ratio in Dietary Supplement on Histopathological Features of the Hippocampus in Experimental Autism Rats}

To confirm whether PUFA at various n-3/n-6 ratios in dietary supplement exerts different neuroprotective effects on the hippocampus in prenatal VPA-exposure rats, Nissl staining was carried out for the evaluation of hippocampal neuronal loss. H\&E staining of control animals revealed that the prefrontal cortex was well-defined and intact and had uniformly 
A

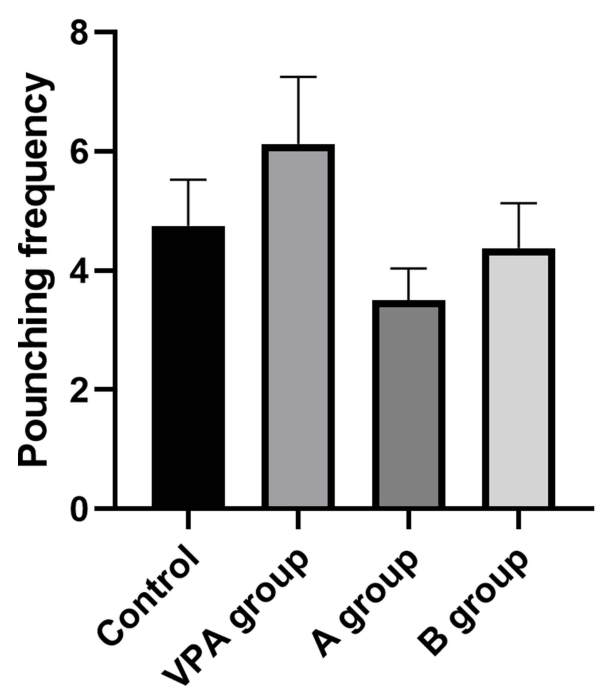

B

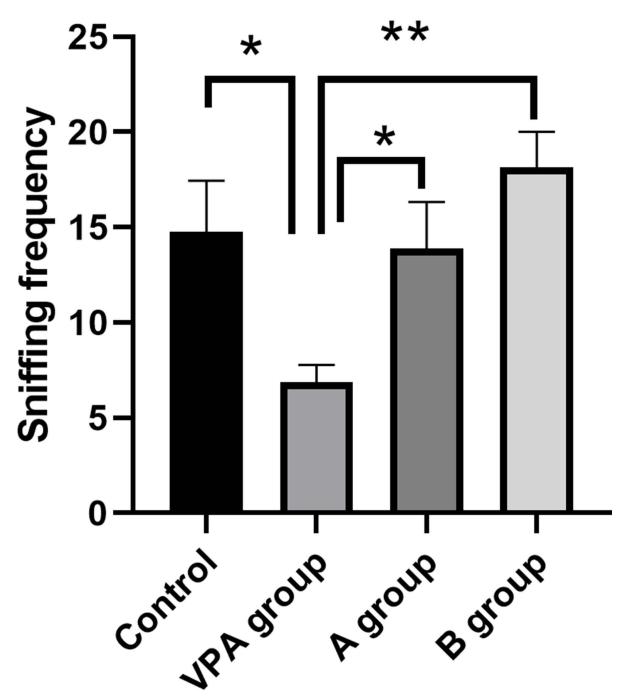

Figure I Effects of n-3/n-6 PUFA (I: 5) supplement on behaviors of VPA rat model. (A and B) effects of n-3/n-6 PUFA (I: 5) supplement on the social play behaviors of the VPAtreated rats. (A) Frequency of pouncing. (B) Frequency of following. Data are expressed as mean \pm SEM.*P<0.05, **P $<0.01$ vs VPA group (One-way ANOVA and LSD test).

sized cells that were orderly arranged without neuronal injury. In the hippocampal CA1 and CA3 regions of prenatal VPA-exposed rats, the disorganization of the external granular and pyramidal layers was observed in the cells which were loosely arranged (Figure 3A). In Nissl staining, neurons normally contain Nissl substance, which are pale blue staining. In the present study, the neuron outlines were clear in control rats, while the structure of cells was compacted with abundant cytoplasm and round cell body. However, in the prenatal VPA-exposure rats, the neuronal loss increased, and neuronal degeneration was observed as shown by sparsely arranged and vague-shaped neurons. The number of eumorphic cells reduced significantly in the prenatal VPA-exposure rats as compared to control rats (Figure 3B). Importantly, n-3/n-6 PUFA (1:5) supplement significantly elevated the density of geomorphic neurons in the prenatal VPA-exposure rats (Figure 3B).

\section{Effects of n-3/n-6 PUFA Ratio in Dietary Supplement on Hippocampal and Serum Inflammatory Biomarkers of Experimental Autism Rats}

In order to investigate the inflammatory response in the brain related to prenatal VPA-exposure, the levels of hippocampal and serum inflammatory cytokines were detected with corresponding ELISA kits. Significant change of hippocampal IL-1 level was observed (Figure 4D, $\mathrm{P}<0.05$ ). The hippocampal IL-1 level increased significantly in the VPA group $(159.695 \pm 17.33 \mathrm{pg} / \mathrm{mL})$ as compared to the control group $(122.14 \pm 1.97 \mathrm{pg} / \mathrm{mL}, \mathrm{P}=0.013)$. The hippocampal IL-1 level in n-3/n-6 PUFA (1:5)-supplemented rats $(130.27 \pm 5.22 \mathrm{pg} / \mathrm{mL})$ decreased markedly as compared to the VPA group $(159.695 \pm 17.33 \mathrm{pg} / \mathrm{mL}, \mathrm{P}<0.05$, Figure 4). The hippocampal IL-6 level increased significantly in the VPA group $(176.06 \pm 20.11 \mathrm{pg} / \mathrm{mL})$ as compared to the control group $(118.25 \pm 4.64 \mathrm{pg} / \mathrm{mL}, \mathrm{P}=0.003$, Figure 4). The hippocampal IL-6 level in n-3/n-6 PUFA (1:5)-supplemented rats $(135.78 \pm 8.88 \mathrm{pg} / \mathrm{mL})$ decreased dramatically as compared to the VPA group $(176.06 \pm 20.11 \mathrm{pg} / \mathrm{mL}, \mathrm{P}=0.03$, Figure 4). The hippocampal TNF- $\alpha$ level increased markedly in the VPA group $(231.24 \pm 8.61 \mathrm{pg} / \mathrm{mL})$ as compared to the control group $(193.4 \pm 5.73 \mathrm{pg} / \mathrm{mL}, \mathrm{P}=0.001)$. The hippocampal TNF- $\alpha$ level in n-3/n-6 PUFA (1:5)-supplemented rats $(200.28 \pm 5.59 \mathrm{pg} / \mathrm{mL})$ decreased significantly as compared to the VPA group $(231.24 \pm 8.61 \mathrm{pg} / \mathrm{mL}, \mathrm{P}=0.003$, Figure 4$)$. However, the changes in the inflammatory cytokine levels were comparable between different groups (Figure 4A-C). 


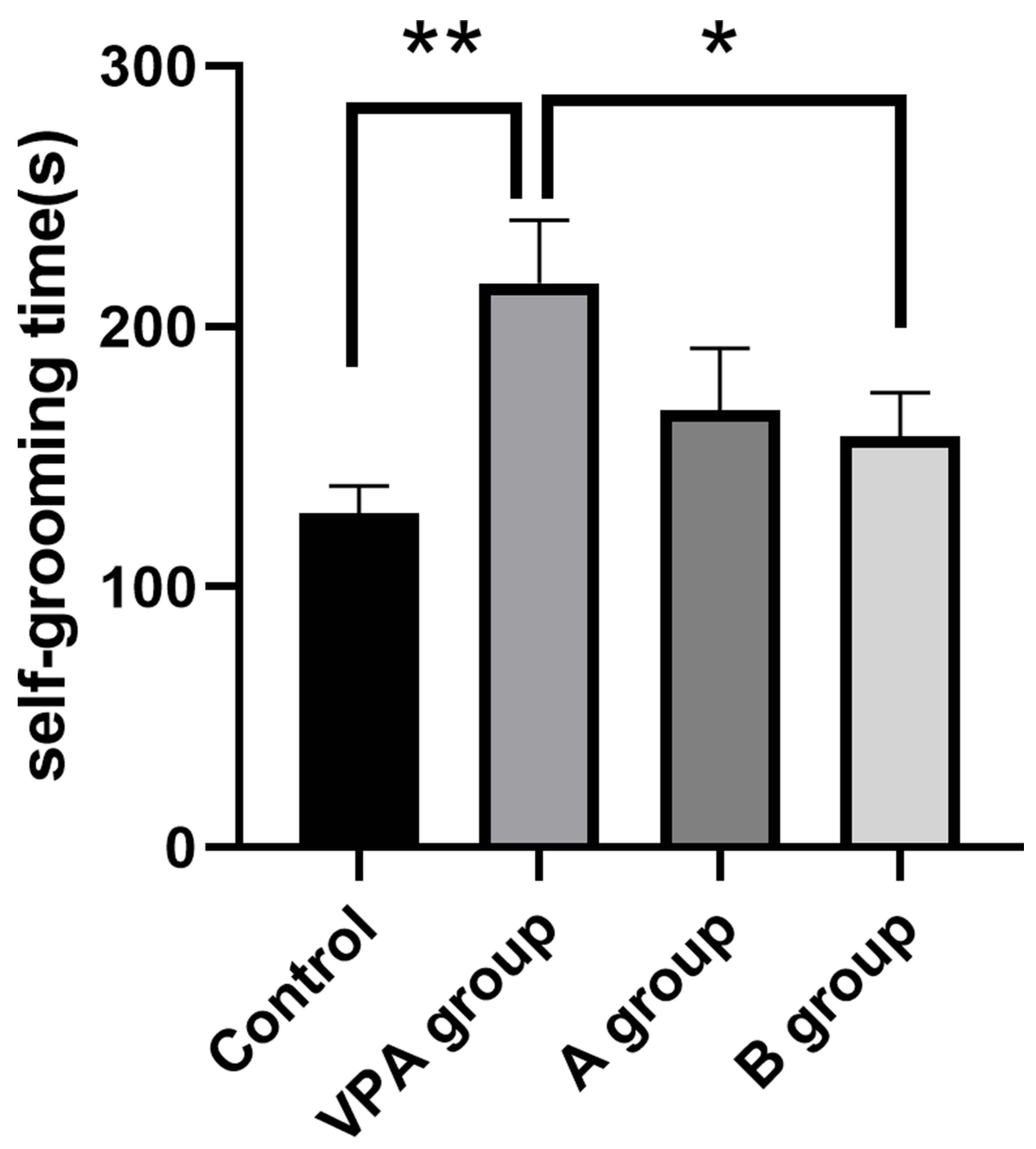

Figure 2 Accumulative time for self-grooming of prenatal VPA-exposed rats decreased following n-3/n-6 PUFA (1:5) supplement. Data are expressed as mean \pm SEM. $* \mathrm{P}<0.05$ vs VPA group, ${ }^{*} \mathrm{P}<0.01$ vs VPA group (One-way ANOVA and LSD test).
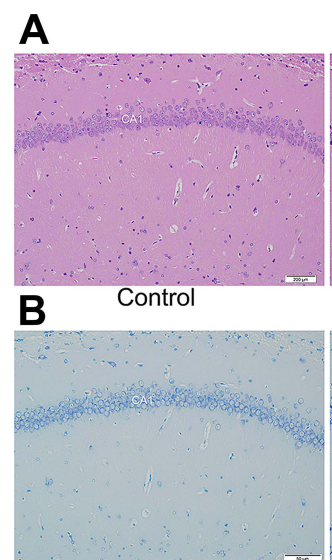

Control

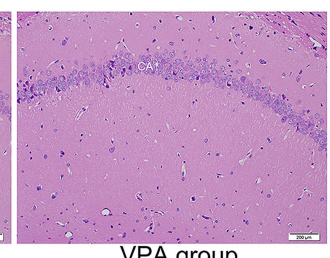

VPA group

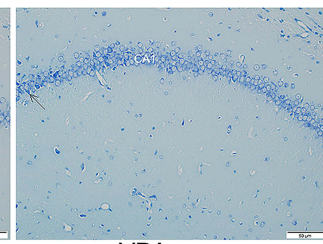

VPA group

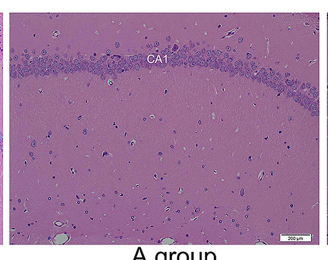

A group

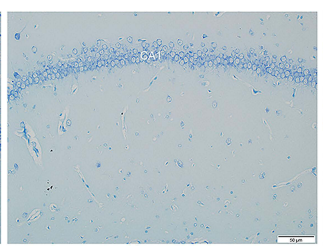

A group

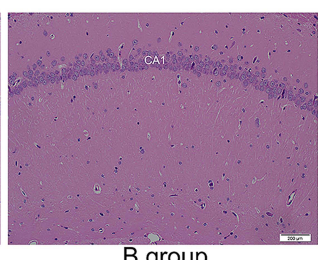

B group

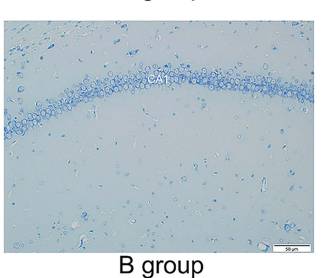

B group

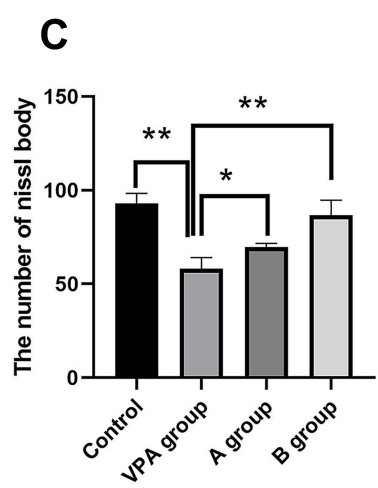

Figure 3 Apoptosis rate and neuronal density in the hippocampal CAI region of VPA rats and rats receiving dietary n-3/n-6 PUFA supplement. (A) Pathological changes of the hippocampus in VPA rats receiving dietary n-3/n-6 PUFA supplement (hematoxylin and eosin staining). (B) Apoptotic neurons on Nissl staining. Arrow: apoptotic cells. (C) The number of Nissl body in hippocampus. $* \mathrm{P}<0.05$, $* * \mathrm{P}<0.01$, compared with VPA group. 

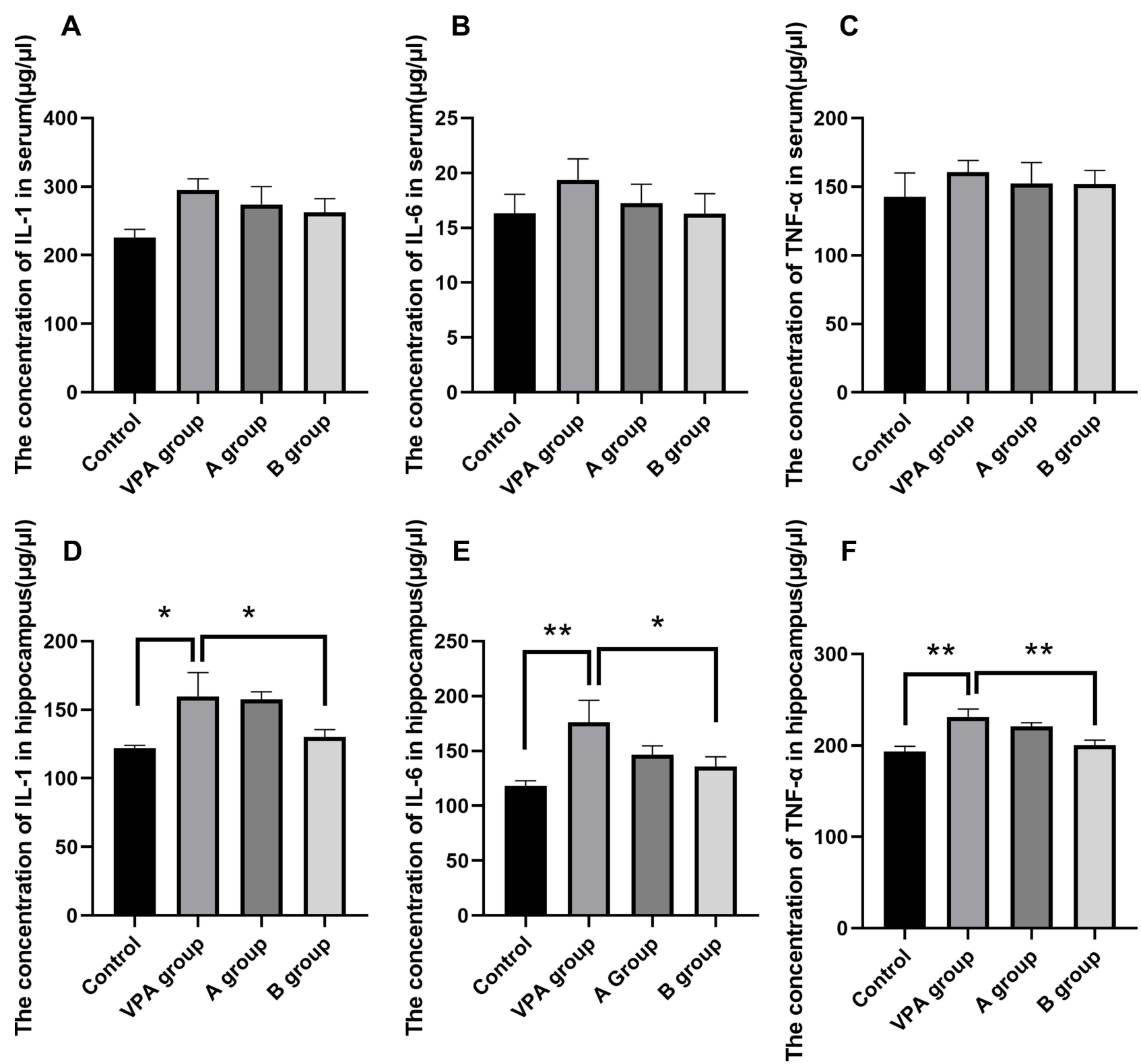

Figure 4 Levels of IL-I, IL-6 and TNF- $\alpha$ in the hippocampus and serum. (A-C) Levels of IL-I, IL-6 and TNF- $\alpha$ in hippocampus; (D-F) Levels of IL-I, IL-6 and TNF- $\alpha$ in the serum. $* \mathrm{P}<0.05$ vs VPA group, ${ }^{* * P}<0.01$ vs VPA group. (One-way ANOVA and LSD tests).

\section{STAT3 Expression in Hippocampus}

The hippocampal tissues were collected from each rat for the detection of STAT3 by Western blotting. Results showed the STAT3 protein expression in rats fed with n-3/n-6 PUFA (1:5) (0.1239 \pm 0.033$)$ decreased significantly as compared to the VPA rats $(0.50 \pm 0.024, \mathrm{P}<0.01, \mathrm{n}=3$, Figure 5$)$. However, the STAT3 protein expression in the hippocampus of VPA rats increased markedly as compared to the control rats $(0.21 \pm 0.064, \mathrm{P}<0.01$, Figure 5$)$.

\section{Discussion}

This study investigated the effects of dietary supplements with PUFA at different $n-3 / n-6$ ratios on the behaviors in a rat model of ASD and the potential molecular mechanism was explored. Our results showed the prenatal VPAexposure-induced social behavior defects, exploratory behaviors and repetitive behaviors were significantly attenuated in rats with n-3/n-6 PUFA (1:5) supplement. Further investigation indicated that the hippocampal levels of IL-6 
A

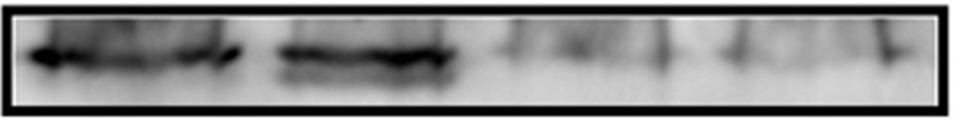

\section{STAT3}

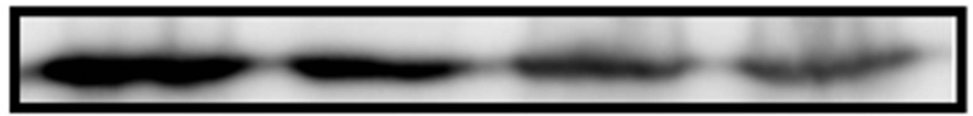

\section{GAPDH}

\section{CONTROL VPA A group B group}
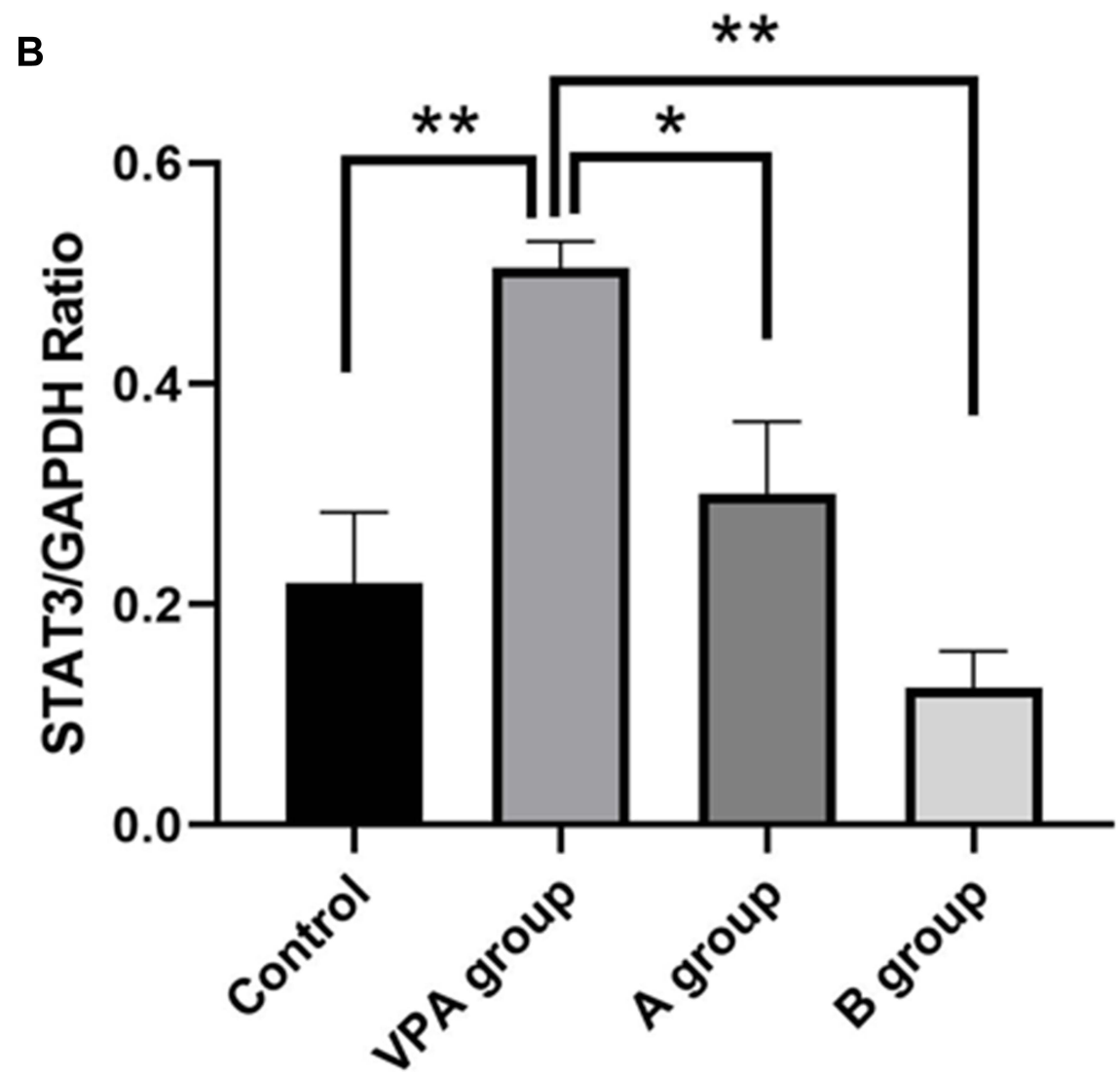

Figure 5 Western blotting of STAT3 protein in the hippocampus. (A) Representative blots of each protein. (B) The level of STAT3 protein was quantified and normalized to GAPDH level. $* \mathrm{P}<0.05$ vs VPA group, $* * \mathrm{P}<0.01$ vs VPA group. (One-way ANOVA and LSD tests).

and TNF- $\alpha$ reduced dramatically in the $n-3 / n-6$ PUFA (1:5) treated rats as compared to VPA treated rats. At the same time, the STAT3 protein expression decreased markedly in the hippocampus of n-3/n-6 PUFA (1:5)-treated rats as compared to the VPA treated rats.

In clinical studies, the maternal use of VPA during pregnancy has been reported to cause abnormalities in the development of fetus and this detrimental effect continues even after birth. These effects include structural malformations and subtle autistic-like behaviors. As a result, prenatal VPA exposure has been proposed as an important risk factor of ASD. ${ }^{19}$ Currently, rodent model of ASD induced by VPA exposure has been widely used in the study of such disorder. ${ }^{20}$ Rats in the ASD model have impaired sociability. ${ }^{21}$ Consistently, in our study, VPA treatment at E12.5 was able to induce defects in the social and explore behaviors, and cause repetitive stereotyped behaviors.

For more than a decade, inflammatory mechanisms have been proposed as a critical contributor to the development of $\mathrm{ASD}^{22}$ Not only inflammation affects the systemic immunities ${ }^{23}$ but neuroinflammation has a robust association with 


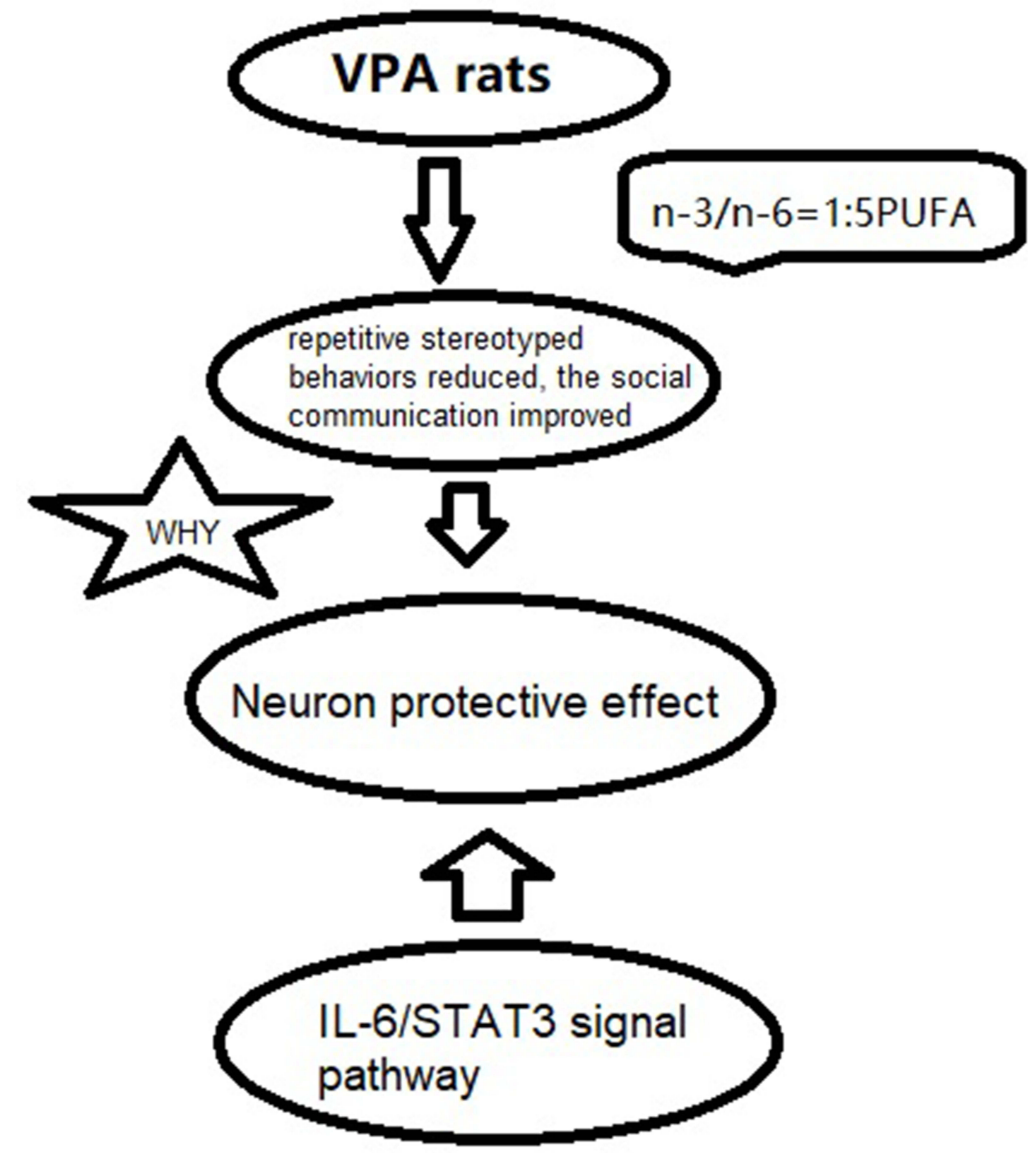

Figure $6 \mathrm{~A}$ scheme of the possible biological pathways.

ASD in patients. In particular, the increased proinflammatory cytokine IL-6 has been reported in the blood samples of ASD children. ${ }^{24}$

Increased IL-6 level in the CNS has been found to be related to multiple neurological diseases caused by brain injury or neuroinflammation. VPA exposure increases the IL-6 level in the brain, potentially through inducing neuroinflammation and altering immune response during neurodevelopment. ${ }^{25}$ Our results also showed significantly increased levels of IL- 6 and TNF- $\alpha$ in the hippocampal tissues of prenatal VPA-exposed rats.

Studies have indicated the direct effects of increased IL- 6 and TNF- $\alpha$ on the neuronal functions and development. ${ }^{26}$ Proinflammatory cytokines IL-6 and TNF- $\alpha$ are also closely related to brain injuries. Jiang et al found that the increased expression of IL-1 $\beta$, IL-6, TNF $\alpha$ and inducible nitric oxide synthase (iNOS) is associated with brain injuries. ${ }^{27}$ The hippocampus is impaired in animal models of $\mathrm{ASD}^{28}$ It has been reported that neuron loss and neurodegeneration (evidenced by sparsely arranged neurons) in the hippocampus are associated with the prenatal VPA-exposed rats. ${ }^{29}$ In our study, Nissl staining showed the prenatal VPA-exposure induced damages to hippocampal neurons, which was characterized by the cavitated neurons. Prenatal VPA-treatments caused the development of ASD, and increased proinflammatory cytokines in the CNS. The elevated inflammation level subsequently causes the damages to the hippocampal neurons, suggesting the robust link between hippocampal neuron damage and elevated neuroinflammation.

Multiple studies have been conducted to investigate the influence of DHA on the ASD. Results have indicated that the changes in the $n-6 / n-3$ ratio in diet at early stages of life potentially induce the alterations of brain connection, synaptogenesis, cognitive functions and behaviors, which are closely associated with the symptoms of ASD. ${ }^{30}$ n-3 PUFA may attenuate the inflammatory responses and ameliorate the saturated fatty acid (SFA)-induced insulin resistance (IR); however, n-6 PUFA promotes the damages caused by IR. ${ }^{31}$ Therefore, PUFA containing n-3/n- 6 at different ratios 
in the supplement may exert distinct effects. In a study of Gao et al, results showed DHA supplements not only promoted the learning of ASD rats, but also attenuated the apoptosis of hippocampal neurons through enhancing the anti-oxidative substances. ${ }^{29}$ In this study, results showed the behavioral improvements of ASD rats with the $n-3 / n-6$ PUFAs (1:5) supplement. Most importantly, n-3/n-6 PUFAs (1:5) supplement was able to attenuate hippocampal neuronal loss and neuroinflammation. The results suggest that $n-3 / n-6$ PUFAs (1:5) supplement is able to attenuate the behavioral impairment of ASD rats and prevent the neuronal death through alleviating neuroinflammation.

During neurodevelopment, IL-6 activates the signal transducer and activator of transcription-3 (STAT3) pathway, which involves in the homeostasis maintaining in neuro- and gliogenesis. ${ }^{32}$ Recently, STAT3 has been suggested to be a key component in the regulation of inflammation. In order to investigate the mechanisms underlying the effects of $n-3 / n-6$ PUFAs (1:5) supplement on the ASD, Western blotting was performed to investigate the changes in the expression of STAT3 (a transcription factor). There is evidence showing that the activation of STAT3 enhances neuroinflammation. ${ }^{33}$ Glycogen synthase kinase $3 \beta$ (GSK3 $\beta$ ) is involved in the regulation of IL-6 release through activating STAT3. ${ }^{34}$ Parker-Athill et al found that IL-6 induced the phosphorylation of Janus Kinase 2 (JAK2)/STAT3, which is related to the functions of IL-6 and closely related to the maternal immune activation-mediated pathological effects. The inhibition of JAK2/STAT3 phosphorylation has also been found to attenuate both behavioral deficits and inflammation. ${ }^{35}$ Parker-Athill et al also found that DIOSMIN was able to modulate the phosphorylation of JAK2/STAT3, which exerted the potential effects on the abnormal autistic-like social behaviors in IL-6/MIA offspring. In this study, higher level of STAT3 was also observed in the hippocampal tissues of ASD rats, and therefore we speculate that the elevated IL-6 level may be related to the activation of STAT3. Meanwhile, rats with dietary supplement of n-3/ n-6 PUFAs (1:5) showed significantly attenuated activation of STAT3 in the hippocampus. Based on the above findings, we postulate that the dietary supplements of $n-3 / n-6$ PUFAs (1:5) is able to attenuate the behavioral deflects in ASD rats via inhibiting STAT3 activation, subsequently leading to the reduced production of IL-6 (Figure 6).

Available studies have revealed that VPA treated mice and monkeys exhibit structural and functional abnormalities in the thalamus, especially the pulvinar nucleus. ${ }^{36,37}$ In fact, pulvinar nucleus is mutually connected with the sensory and prefrontal cortex and plays important roles in the contextual and multi-sensory processing, ${ }^{38-40}$ which is dysfunctional in autistic animals and patients. Thus, the pulvinar nucleus may be involved in the VPA induced autistic characteristics. Therefore, further studies are needed to examine the molecular and cellular changes in the pulvinar nucleus of VPA treated animals.

\section{Conclusion}

This study for the first time investigates the alterations of the hippocampus in a rat model of ASD, and provides novel insight to the role of neuroinflammation in the altered behaviors of VPA treated rats. Additionally, n-3/n-6 PUFAs (1:5) supplement exhibits anti-inflammatory effects by inhibiting STAT3 activation, suppressing the release of downstream proinflammatory cytokines in the ASD rats, and reducing the apoptosis of hippocampal neurons, which subsequently improve the behaviors of ASD rats. Specifically, nutritional interventions, especially those with anti-inflammatory capabilities, are promising candidates for the treatment of ASD due to their potential effects on the neuroinflammation.

\section{Acknowledgments}

This work was supported by the Natural Science Foundation of China (No. 81602847) and Scientific Research

Foundation from Educational Commission of Jilin Province of China (No. JJKH20211165kJ).

\section{Disclosure}

The authors report no conflicts of interest in this work.

\section{References}

1. Chen JA, Penagarikano O, Belgard TG, Swarup V, Geschwind DH. The emerging picture of autism spectrum disorder: genetics and pathology. Annu Rev Pathol. 2015;10:111-144. doi:10.1146/annurev-pathol-012414-040405

2. Roullet FI, Lai JK, Foster JA. In utero exposure to valproic acid and autism-a current review of clinical and animal studies. Neurotoxicol Teratol. 2013;36:47-56. doi:10.1016/j.ntt.2013.01.004

3. Markram K, Rinaldi T, La Mendola D, Sandi C, Markram H. Abnormal fear conditioning and amygdala processing in an animal model of autism. Neuropsychopharmacology. 2008;33(4):901-912. doi:10.1038/sj.npp.1301453 
4. Rodier PM, Ingram JL, Tisdale B, Nelson S, Romano J. Embryological origin for autism: developmental anomalies of the cranial nerve motor nuclei. J Comp Neurol. 1996;370(2):247-261. doi:10.1002/(SICI)1096-9861(19960624)370:2<247::AID-CNE8>3.0.CO;2-2

5. Al-Amin MM, Rahman MM, Khan FR, Zaman F, Mahmud Reza H. Astaxanthin improves behavioral disorder and oxidative stress in prenatal valproic acid-induced mice model of autism. Behav Brain Res. 2015;286:112-121. doi:10.1016/j.bbr.2015.02.041

6. Xu N, Li X, Zhong Y. Inflammatory cytokines: potential biomarkers of immunologic dysfunction in autism spectrum disorders. Mediators Inflamm. 2015;2015:531518. doi:10.1155/2015/531518

7. Bazinet RP, Laye S. Polyunsaturated fatty acids and their metabolites in brain function and disease. Nat Rev Neurosci. 2014;15(12):771-785. doi:10.1038/nrn3820

8. Parletta N, Milte CM, Meyer BJ. Nutritional modulation of cognitive function and mental health. J Nutr Biochem. 2013;24(5):725-743. doi:10.1016/j.jnutbio.2013.01.002

9. Simopoulos AP. The importance of the ratio of omega-6/omega-3 essential fatty acids. Biomed Pharmacother. 2002;56(8):365-379. doi:10.1016/ S0753-3322(02)00253-6

10. Brown CM, Austin DW. Autistic disorder and phospholipids: a review. Prostaglandins Leukot Essent Fatty Acids. 2011;84(1-2):25-30. doi:10.1016/j.plefa.2010.09.007

11. Innis SM, de La Presa Owens S. Dietary fatty acid composition in pregnancy alters neurite membrane fatty acids and dopamine in newborn rat brain. J Nutr. 2001;131(1):118-122. doi:10.1093/jn/131.1.118

12. Keim SA, Gracious B, Boone KM, et al. omega-3 and omega- 6 fatty acid supplementation may reduce autism symptoms based on parent report in preterm toddlers. J Nutr. 2018;148(2):227-235. doi:10.1093/jn/nxx047

13. Puri BK, Martins JG. Which polyunsaturated fatty acids are active in children with attention-deficit hyperactivity disorder receiving PUFA supplementation? A fatty acid validated meta-regression analysis of randomized controlled trials. Prostaglandins Leukot Essent Fatty Acids. 2014;90(5):179-189. doi:10.1016/j.plefa.2014.01.004

14. Wang JP, Xu YC, Hou JQ, et al. Effects of dietary fat profile on gut microbiota in valproate animal model of autism. Front Med (Lausanne). 2020;7:151. doi:10.3389/fmed.2020.00151

15. Melancia F, Servadio M, Schiavi S, Campolongo P, Giusti-Paiva A, Trezza V. Testing the correlation between experimentally-induced hypothyroidism during pregnancy and autistic-like symptoms in the rat offspring. Behav Brain Res. 2017;321:113-122. doi:10.1016/j.bbr.2016.12.032

16. Panksepp J, Siviy S, Normansell L. The psychobiology of play: theoretical and methodological perspectives. Neurosci Biobehav Rev. 1984;8 (4):465-492. doi:10.1016/0149-7634(84)90005-8

17. Kalueff AV, Stewart AM, Song C, Berridge KC, Graybiel AM, Fentress JC. Neurobiology of rodent self-grooming and its value for translational neuroscience. Nat Rev Neurosci. 2016;17(1):45-59. doi:10.1038/nrn.2015.8

18. Hill MN, Karatsoreos IN, Hillard CJ, McEwen BS. Rapid elevations in limbic endocannabinoid content by glucocorticoid hormones in vivo. Psychoneuroendocrinology. 2010;35(9):1333-1338. doi:10.1016/j.psyneuen.2010.03.005

19. Christensen J, Gronborg TK, Sorensen MJ, et al. Prenatal valproate exposure and risk of autism spectrum disorders and childhood autism. JAMA. 2013;309(16):1696-1703. doi:10.1001/jama.2013.2270

20. Tartaglione AM, Schiavi S, Calamandrei G, Trezza V. Prenatal valproate in rodents as a tool to understand the neural underpinnings of social dysfunctions in autism spectrum disorder. Neuropharmacology. 2019;159:107477. doi:10.1016/j.neuropharm.2018.12.024

21. Win-Shwe TT, Nway NC, Imai M, Lwin TT, Mar O, Watanabe H. Social behavior, neuroimmune markers and glutamic acid decarboxylase levels in a rat model of valproic acid-induced autism. J Toxicol Sci. 2018;43(11):631-643. doi:10.2131/jts.43.631

22. Estes ML, McAllister AK. Maternal TH17 cells take a toll on baby's brain. Science. 2016;351(6276):919-920. doi:10.1126/science.aaf2850

23. Goines P, Van de Water J. The immune system's role in the biology of autism. Curr Opin Neurol. 2010;23(2):111-117. doi:10.1097/ WCO.0b013e3283373514

24. Zerbo O, Yoshida C, Grether JK, et al. Neonatal cytokines and chemokines and risk of Autism Spectrum Disorder: the Early Markers for Autism (EMA) study: a case-control study. J Neuroinflammation. 2014;11:113. doi:10.1186/1742-2094-11-113

25. Al-Askar M, Bhat RS, Selim M, Al-Ayadhi L, El-Ansary A. Postnatal treatment using curcumin supplements to amend the damage in VPA-induced rodent models of autism. BMC Complement Altern Med. 2017;17(1):259. doi:10.1186/s12906-017-1763-7

26. Ashwood P, Nguyen DV, Hessl D, Hagerman RJ, Tassone F. Plasma cytokine profiles in Fragile X subjects: is there a role for cytokines in the pathogenesis? Brain Behav Immun. 2010;24(6):898-902. doi:10.1016/j.bbi.2010.01.008

27. Jiang Q, Chen J, Long X, et al. Phillyrin protects mice from traumatic brain injury by inhibiting the inflammation of microglia via PPARgamma signaling pathway. Int Immunopharmacol. 2020;79:106083. doi:10.1016/j.intimp.2019.106083

28. Edgin JO, Pennington BF. Spatial cognition in autism spectrum disorders: superior, impaired, or just intact? J Autism Dev Disord. 2005;35(6):729745. doi:10.1007/s10803-005-0020-y

29. Gao J, Wang X, Sun H, et al. Neuroprotective effects of docosahexaenoic acid on hippocampal cell death and learning and memory impairments in a valproic acid-induced rat autism model. Int J Dev Neurosci. 2016;49:67-78. doi:10.1016/j.ijdevneu.2015.11.006

30. van Elst K, Bruining H, Birtoli B, Terreaux C, Buitelaar JK, Kas MJ. Food for thought: dietary changes in essential fatty acid ratios and the increase in autism spectrum disorders. Neurosci Biobehav Rev. 2014;45:369-378. doi:10.1016/j.neubiorev.2014.07.004

31. Liu HQ, Qiu Y, Mu Y, et al. A high ratio of dietary n-3/n-6 polyunsaturated fatty acids improves obesity-linked inflammation and insulin resistance through suppressing activation of TLR4 in SD rats. Nutr Res. 2013;33(10):849-858. doi:10.1016/j.nutres.2013.07.004

32. He F, Ge W, Martinowich K, et al. A positive autoregulatory loop of Jak-STAT signaling controls the onset of astrogliogenesis. Nat Neurosci. 2005;8(5):616-625. doi:10.1038/nn1440

33. D’Angelo B, Ek CJ, Sun Y, Zhu C, Sandberg M, Mallard C. GSK3beta inhibition protects the immature brain from hypoxic-ischaemic insult via reduced STAT3 signalling. Neuropharmacology. 2016;101:13-23. doi:10.1016/j.neuropharm.2015.09.017

34. Beurel E, Jope RS. Lipopolysaccharide-induced interleukin-6 production is controlled by glycogen synthase kinase-3 and STAT3 in the brain. $J$ Neuroinflammation. 2009;6:9. doi:10.1186/1742-2094-6-9

35. Parker-Athill E, Luo D, Bailey A, et al. Flavonoids, a prenatal prophylaxis via targeting JAK2/STAT3 signaling to oppose IL-6/MIA associated autism. J Neuroimmunol. 2009;217(1-2):20-27. doi:10.1016/j.jneuroim.2009.08.012

36. Hu Y, Chen Z, Huang L, et al. A translational study on looming-evoked defensive response and the underlying subcortical pathway in autism. Sci Rep. 2017;7(1):14755. doi:10.1038/s41598-017-15349-x 
37. Tang Y, Yu X, Zhang X, et al. Single-dose intravenous administration of antiepileptic drugs induces rapid and reversible remodeling in the brain: evidence from a voxel-based morphometry evaluation of valproate and levetiracetam in rhesus monkeys. Neuroscience. 2015;303:595-603. doi:10.1016/j.neuroscience.2015.07.039

38. Chou XL, Fang Q, Yan L, et al. Contextual and cross-modality modulation of auditory cortical processing through pulvinar mediated suppression. Elife. 2020;9. doi:10.7554/eLife.54157

39. Fang Q, Chou XL, Peng B, Zhong W, Zhang LI, Tao HW. A differential circuit via retino-colliculo-pulvinar pathway enhances feature selectivity in visual cortex through surround suppression. Neuron. 2020;105(2):355-369.e356. doi:10.1016/j.neuron.2019.10.027

40. Ibrahim LA, Mesik L, Ji XY, et al. Cross-modality sharpening of visual cortical processing through layer-1-mediated inhibition and disinhibition. Neuron. 2016;89(5):1031-1045. doi:10.1016/j.neuron.2016.01.027

\section{Publish your work in this journal}

Neuropsychiatric Disease and Treatment is an international, peer-reviewed journal of clinical therapeutics and pharmacology focusing on concise rapid reporting of clinical or pre-clinical studies on a range of neuropsychiatric and neurological disorders. This journal is indexed on PubMed Central, the 'PsycINFO' database and CAS, and is the official journal of The International Neuropsychiatric Association (INA). The manuscript management system is completely online and includes a very quick and fair peer-review system, which is all easy to use. Visit http://www.dovepress.com/testimonials.php to read real quotes from published authors.

Submit your manuscript here: https://www.dovepress.com/neuropsychiatric-disease-and-treatment-journal 